\title{
Crescimento e produção de gérbera fertirrigada com solução nutritiva
}

\section{Fernanda Ludwig; Dirceu M Fernandes; Poliana RD Mota; Roberto L Villas Bôas}

UNESP-FCA, Dep ${ }^{\text {to }}$ Recursos Naturais, C. Postal 237, 18610-307 Botucatu-SP; ludwig.fernanda@yahoo.com.br; dmfernandes@fca. unesp.br; polimota@yahoo.com.br; rlvboas@fca.unesp.br

\section{RESUMO}

Este trabalho foi conduzido com o objetivo de avaliar as características de crescimento e produção de quatro cultivares de gérbera de vaso fertirrigadas com duas soluções nutritivas. O experimento foi realizado em casa-de-vegetação, durante o período de maio a julho de 2006, na UNESP-FCA em Botucatu-SP. O delineamento experimental foi de blocos casualizados e constituído em esquema fatorial $4 \times 2$, sendo 4 cultivares de gérbera (Cherry, Golden Yellow, Salmon Rose e Orange) e 2 soluções nutritivas. As plantas foram conduzidas em vasos com volume de 1,3 L, preenchidos com substrato composto de $70 \%$ de casca de pinus fina e $30 \%$ de terra de subsolo. Avaliouse o número de folhas e diâmetro de planta semanalmente durante o ciclo da cultura; área foliar e fitomassa seca ao final dos períodos vegetativo e reprodutivo; tempo necessário para a produção, número de inflorescências, diâmetro das inflorescências e hastes e altura de planta, no ponto de comercialização. As cultivares responderam de maneira diferenciada quanto às características de crescimento, Salmon Rose e Cherry apresentaram maior formação de parte aérea; Golden Yellow e Orange foram mais compactas. No ponto de comercialização, somente altura, diâmetro de inflorescência e haste apresentaram variação entre as cultivares. As duas soluções nutritivas foram eficientes para a produção de plantas de qualidade.

Palavras-chave: Gerbera jamesonii, floricultura, fertirrigação, nutrição de plantas.

\author{
ABSTRACT \\ Growth and production of gerbera fertigated with nutrient \\ solution
}

This work aimed to evaluate growth and production characteristics of four potted gerbera cultivars fertigated with two nutrient solutions. The experiment was carried out in a greenhouse from May to July 2006 at UNESP, Botucatu, São Paulo State, Brazil. Experimental design was in randomized blocks in a $4 \times 2$ factorial arrangement, with 4 gerbera cultivars (Cherry, Golden Yellow, Salmon Rose and Orange) and 2 nutrient solutions. The plants were cultivated in $1.3 \mathrm{~L}$ pots filled with substrate composed of $70 \%$ fine pinus bark and $30 \%$ subsoil earth. Leaf number and plant diameter were weekly evaluated throughout the culture cycle; leaf area and dry phytomass at the end of vegetative and reproductive periods; and time needed for production, inflorescence number, inflorescence diameter, and plant stem and height at commercialization point. The cultivars presented different responses to growth characteristics: Salmon Rose and Cherry presented higher shoot formation; Golden Yellow and Orange were more compact. At commercialization point, only height, inflorescence diameter and stem varied among cultivars. Both nutrient solutions were effective to produce high-quality plants.

Keywords: Gerbera jamesonii, floriculture, fertigation, plant nutrition.

\section{(Recebido para publicação em 26 de março de 2009; aceito em 1 de julho de 2010) (Received on March 26, 2009; accepted on July 1, 2010)}

\begin{abstract}
$\mathrm{A}$ gérbera é uma planta herbácea, pertencente à família Asteraceae e originária da África do Sul. As folhas têm formato em roseta e do pecíolo de algumas delas evoluem os brotos florais que desenvolvem pedúnculos com inflorescência terminal em capítulo (Infoagro, 2005). Segundo Bellé (1998), o comprimento do pedúnculo floral varia de acordo com a cultivar, sendo longo para corte, e compacto para vaso.

As espécies selvagens de Gerbera jamesonii e as cultivares desenvolvidas inicialmente apresentavam hastes longas e eram mais adaptadas para uso de flor de corte que de vaso. A primeira cultivar selecionada para vaso foi introduzida no Japão, no início de 1980, representando importante avanço no desenvolvimento da cultura (Rojers \& Tjia, 1990).
\end{abstract}

Durante o processo de seleção das cultivares, as principais características consideradas são a cor e forma das inflorescências. Entretanto, existem variações quanto ao desenvolvimento em diferentes condições de cultivo e ambiente. $\mathrm{O}$ desempenho de cultivares de gérbera bem como as características morfológicas e genéticas vêm sendo avaliados em alguns trabalhos (Chung $e t$ al., 2001; Singh \& Mandhar, 2001), nos quais muitas variações estão relacionadas às peculiaridades genéticas.

Esta espécie tem sido mais amplamente estudada como flor de corte, tendo reduzidas informações disponíveis sobre as técnicas de cultivo como flor de vaso, tanto internacional, como nacionalmente, e desse modo, o manejo dos fatores de produção muitas vezes é realizado de maneira empírica pelos produtores. Dentre estes, destaca-se a adubação e nutrição, por empregar grandes quantidades de nutrientes, que por vezes não é demandada pela cultura, ocorrendo assim um aumento da salinidade do substrato e consequentemente diminuindo a qualidade de produção.

A utilização da fertirrigação tem aumentado no setor produtivo de flores e plantas ornamentais, onde se busca principalmente a máxima qualidade do produto final. A aplicação de nutrientes via solução nutritiva apresenta vantagem da manutenção das concentrações preconizadas dos nutrientes durante o período de desenvolvimento da planta (Barbosa et al., 1999). O rígido controle de sais disponíveis no substrato torna-se de extrema importância, pois em condições de salinidade, as plantas respondem com redução do crescimento, embora algumas plantas apresentem variação 
quanto à tolerância à salinidade.

Nesse sentido, algumas pesquisas vêm sendo desenvolvidas visando à avaliação de soluções nutritivas com diferentes condutividades elétricas no desenvolvimento de flores envasadas. Para a cultura da gérbera, autores afirmam que a condutividade elétrica de $1,0 \mathrm{dS} \mathrm{m}^{-1}$ aplicada via subirrigação para a cultivar Shogun (Zheng et al., 2004) e de $3,5 \mathrm{dS} \mathrm{m}^{-1}$ via fertirrigação por gotejo, para as cultivares Cherry e Salmon Rose (Mota, 2007) foram adequadas ao desenvolvimento de plantas com qualidade satisfatória. Soluções com condutividade elétrica superior a 2,57 $\mathrm{dS} \mathrm{m}^{-1}$ aplicadas à cultura do crisântemo por Mota (2004), promoveram redução na área foliar, devido à maior cerosidade e espessura promovida pelo estresse salino.

Busca-se, para gérbera cultivada em vaso, um elevado número de hastes florais com grande diâmetro das inflorescências, porte baixo, bem como uma adequada ramificação de folhas, formando um conjunto equilibrado entre a parte vegetativa e reprodutiva. Esta pesquisa foi desenvolvida com o objetivo de avaliar as características de crescimento e produção em quatro cultivares de gérbera (Gerbera jamesonii), fertirrigadas com duas soluções nutritivas.

\section{MATERIAL E MÉTODOS}

O experimento foi conduzido de maio a julho de 2006, em casa-devegetação da UNESP-FCA, município de Botucatu (22 $51^{\prime}$ 'S; 48²6' W; 830 m de altitude), estado de São Paulo.

A casa-de-vegetação consta de estrutura de teto em arco, com laterais de tela branca, pavimentada com concreto, em uma área total de $168 \mathrm{~m}^{2}$ (7 x 24 m) e 2,6 $\mathrm{m}$ de pé direito. Objetivando conduzir a cultura numa intensidade luminosa inferior a 50.000 lux, instalou-se na parte superior da estrutura, uma malha termorefletora com 50\% (Aluminet ${ }^{\circledR}$ ), a qual permanecia fechada das 10:30 às 16:00 horas e aberta nos demais horários. A temperatura média no interior da casade-vegetação foi de $20,5^{\circ} \mathrm{C}$ e a umidade relativa média do ar foi de $69 \%$.

Adotou-se o delineamento experi- mental em blocos casualizados, empregando o esquema fatorial $4 \times 2$ (quatro cultivares e duas soluções nutritivas), em cinco repetições e seis plantas por parcela. As cultivares utilizadas são provenientes do grupo Festival, da empresa Sakata Seed Sudamerica, de grande aceitação no mercado, sendo Cherry e Golden Yellow, pertencentes à série Dark Eyes (centro escuro), e Salmon Rose e Orange, pertencentes à série Light Eyes (centro claro).

As soluções nutritivas diferiram em relação à condutividade elétrica (CE), sendo que a mais concentrada apresentou $1,76 \mathrm{dS} \mathrm{m}^{-1}\left(\mathrm{~S}_{2}\right)$ e a seguinte

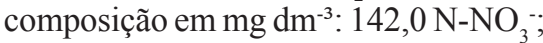
101,5 N-NH ${ }_{4}^{+}$; 105,0 K; 25,2 P; 51,3 Ca; 6,3 Mg; 28,0 S; 0,2 B; 0,3 Cu; 3,9 Fe; 1,4 $\mathrm{Mn} ; 0,1 \mathrm{Mo} \mathrm{e} 0,3 \mathrm{Zn}$, no período vegetativo. A solução com $\mathrm{CE}$ de $0,92 \mathrm{dS} \mathrm{m} \mathrm{m}^{-1}\left(\mathrm{~S}_{1}\right)$ apresentava metade da concentração de sais da $\mathrm{S}_{2}$. Estas foram reformuladas quando a planta entrou na fase reprodutiva, aos 41 dias após a aclimatação (DAA), devido à mudança na demanda da cultura, tendo a $\mathrm{S}_{2}$ uma $\mathrm{CE}$ de 2,04 $\mathrm{dS} \mathrm{m} \mathrm{m}^{-1}$ e a seguinte composição em $\mathrm{mg}$ $\mathrm{dm}^{-3}: 110,3{\mathrm{~N}-\mathrm{NO}_{3}^{-}}^{-}, 66,8 \mathrm{~N}^{-N_{4}}{ }_{4}^{+} ; 285,0$ $\mathrm{K} ; 56,6 \mathrm{P} ; 26,3 \mathrm{Ca} ; 17,18 \mathrm{Mg} ; 76 \mathrm{~S} ; 0,4$ B; 0,4 Cu; 4,4 Fe;1,7 Mn; 0,1 Mo e 0,5 $\mathrm{Zn}$. A exemplo do que ocorreu para a fase vegetativa a solução com $\mathrm{CE}$ de $1,07 \mathrm{dS} \mathrm{m}^{-1}\left(\mathrm{~S}_{1}\right)$ apresentou metade da concentração de sais.

As plantas receberam os nutrientes via fertirrigação, aplicados manualmente uma vez ao dia. A lâmina de irrigação correspondeu à quantidade de água requerida, a fim de elevar a umidade do substrato contido no vaso ao valor correspondente à condição de máxima retenção (Fanela et al., 2006). A quantidade média de solução nutritiva fornecida para as cultivares Golden Yellow e Orange foi de $100 \mathrm{~mL} / \mathrm{vaso} / \mathrm{dia}$ nos períodos vegetativo e reprodutivo, $\mathrm{e}$ para as cultivares Cherry e Salmon Rose foi de $100 \mathrm{~mL} / \mathrm{vaso} / \mathrm{dia}$ no período vegetativo e $150 \mathrm{~mL} / \mathrm{vaso} /$ dia no período reprodutivo.

Para a instalação do experimento foram utilizadas mudas de Gerbera jamesonii, para cultivo em vaso, com tratamento prévio de aclimatação, durante um período de 30 dias. Estas foram conduzidas em vasos com volume de
1,3 L e dimensões de 12,2 cm de altura, $14,8 \mathrm{~cm}$ de base superior e $9,8 \mathrm{~cm}$ de base inferior, preenchidos com substrato composto de $70 \%$ de casca de pinus fina e $30 \%$ de terra de subsolo.

Semanalmente realizou-se a contagem do número de folhas $(\mathrm{NF})$ e a medida do diâmetro da planta (DP), efetuados em duas plantas por parcela, com os resultados expressos em valor médio. O DP foi medido com o auxílio de régua graduada em milímetros, a partir de duas extremidades opostas da planta, perpendiculares entre si.

As determinações de área foliar (AF) e fitomassa seca total da parte aérea (FS) foram realizadas ao final dos períodos vegetativo (41 DAA) e reprodutivo (62 DAA). A AF foi efetuada em medidor tipo "scanner" modelo Li 3100, da marca Licor. A parte aérea total das plantas foi lavada e posta em estufa de ventilação forçada a $65^{\circ} \mathrm{C}$ por um período médio de 48 horas, até massa constante, a fim de que fosse obtida sua FS.

Durante o crescimento vegetativo, como é padrão nas produções comerciais, foram desbastados os primeiros botões florais desenvolvidos (BD), pois a planta ainda não apresentava conformação foliar adequada para permitir seu desenvolvimento e assim a qualidade das plantas seria afetada. Estes foram retirados até os $30 \mathrm{DAA}$, sendo contabilizados.

O tempo necessário para a produção (TP), em DAA, foi avaliado adotando-se o dia em que as plantas atingiam o ponto de comercialização, o qual é caracterizado pela abertura dos estames, devendo a inflorescência apresentar no mínimo dois círculos destes abertos com liberação de pólen (Lin \& French, 1985).

No ponto de comercialização, as plantas eram avaliadas quanto à altura de planta (AP), número de inflorescências (NI), diâmetro de inflorescências (DI) e diâmetro das hastes florais (DH). A AP foi mensurada com auxílio de régua graduada em milímetros, a partir da parte superior do vaso até a extremidade superior da planta. $\mathrm{O} \mathrm{DH}$ foi determinado aos cinco centímetros abaixo do capítulo floral, com auxílio de paquímetro digital da marca Starret, o qual também foi utilizado para a medida do diâmetro de inflorescência, adotando-se dois pontos 
extremos, perpendiculares entre si.

Os resultados foram submetidos à análise de variância pelo teste $\mathrm{F}$. As médias foram comparadas pelo teste Tukey a 5\% de probabilidade, quando significativos, com o uso do programa estatístico Sisvar (Ferreira, 2000).

\section{RESULTADOS E DISCUSSÃO}

De acordo com os resultados obtidos (Tabelas 1, 2 e 3), as soluções nutritivas somente apresentaram efeito significativo para o número de folhas (NF) aos nove dias após aclimatação
(DAA), quando verificou-se maior desenvolvimento na $S_{1}$, e para o diâmetro de planta (DP) aos 51 DAA, com acréscimo na $\mathrm{S}_{2}$.

As cultivares Cherry e Salmon Rose destacaram-se quanto ao DP e área foliar (AF) (Tabelas 1 e 2). Variações entre cultivares de gérbera também foram registradas por Singh \& Mandhar (2001) para o DP, por Paradiso et al. (2003) e Mota (2007) para a AF, inferindo que essas características são reguladas principalmente pelas particularidades genéticas das cultivares.

Concordando com os resultados obtidos, Zheng et al. (2004), concluíram que a AF não é alterada pela diluição da solução nutritiva, ao avaliarem a aplicação de soluções com CE de 0,2; 0,$5 ; 1,0$ e $1,7 \mathrm{dS} \mathrm{m}^{-1}$ para gérbera de vaso cultivar Shogun. O fornecimento de soluções nutritivas com $\mathrm{CE}$ de 3,5 e $5,0 \mathrm{dS} \mathrm{m}^{-1}$ para as cultivares de gérbera Cherry e Golden Yellow, respectivamente, promoveram maior AF somente aos 42 DAA, em trabalho desenvolvido por Mota (2007).

$\mathrm{O}$ incremento diário para $\mathrm{AF}$ foi superior nos últimos 21 dias para Cherry $\left(2,2 \mathrm{~cm}^{2} \mathrm{dia}^{-1}\right)$ e Salmon Rose $(2,1$ $\left.\mathrm{cm}^{2} \mathrm{dia}^{-1}\right)$ e pouco inferior para Golden Yellow $\left(1,5 \mathrm{~cm}^{2} \mathrm{dia}^{-1}\right)$ e Orange $\left(1,3 \mathrm{~cm}^{2}\right.$

Tabela 1. Número de folhas (NF) e diâmetro de planta (DP), em cultivares de gérbera fertirrigadas com soluções nutritivas (number of leaves (NF) and plant diameter (DP) of gerbera cultivars fertigated with nutritive solution). Botucatu, UNESP, 2006.

\begin{tabular}{|c|c|c|c|c|c|c|c|c|c|}
\hline & \multicolumn{9}{|c|}{ Dias após aclimatação } \\
\hline & 2 & 9 & 16 & 23 & 30 & 37 & 44 & 51 & 58 \\
\hline Cultivar & \multicolumn{9}{|c|}{ Folhas $\left(n^{0}\right)$} \\
\hline Cherry & 8,6 & 10,0 & 11,3 & 13,3 & 16,0 & 18,5 & 21,3 & 24,1 & 26,1 \\
\hline Golden Yellow & 7,8 & 8,8 & 10,1 & 11,7 & 14,0 & 16,6 & 18,7 & 21,1 & 22,3 \\
\hline Salmon Rose & 9,2 & 10,2 & 11,6 & 13,3 & 14,8 & 17,4 & 19,9 & 23,0 & 25,4 \\
\hline Orange & 8,4 & 9,1 & 11,1 & 12,8 & 14,4 & 16,5 & 18,0 & 20,3 & 21,5 \\
\hline \multicolumn{10}{|l|}{ Solução } \\
\hline $\mathrm{S}_{1}$ & 8,8 & $9,9 \mathrm{~A}$ & 11,5 & 13,3 & 15,3 & 17,9 & 19,8 & 22,4 & 23,7 \\
\hline $\mathrm{S}_{2}$ & 8,1 & $9,0 \mathrm{~B}$ & 10,6 & 12,3 & 14,4 & 16,6 & 19,2 & 21,9 & 23,9 \\
\hline $\mathrm{C}$ & ns & ns & $\mathrm{ns}$ & ns & ns & $\mathrm{ns}$ & ns & ns & $\mathrm{ns}$ \\
\hline S & ns & $*$ & ns & ns & ns & ns & ns & ns & ns \\
\hline $\mathrm{C} * \mathrm{~S}$ & $\mathrm{~ns}$ & ns & ns & ns & ns & ns & ns & ns & $\mathrm{ns}$ \\
\hline CV (\%) & 14,8 & 13,1 & 13,3 & 15,4 & 16,2 & 16,7 & 17,1 & 18,0 & 15,6 \\
\hline Cultivar & \multicolumn{9}{|c|}{ Diâmetro da planta (cm) } \\
\hline Cherry & $21,3 \mathrm{a}$ & $23,5 \mathrm{ab}$ & $26,6 \mathrm{a}$ & $28,5 \mathrm{a}$ & $30,0 \mathrm{a}$ & $31,9 \mathrm{a}$ & $32,8 \mathrm{a}$ & $34,3 \mathrm{a}$ & $35,0 \mathrm{a}$ \\
\hline Golden Yellow & $19,3 b$ & $21,4 \mathrm{c}$ & $23,4 b$ & $24,5 \mathrm{c}$ & $26,2 b$ & $27,8 b$ & $29,8 b$ & $31,1 \mathrm{~b}$ & $31,4 b$ \\
\hline Salmon Rose & $21,5 \mathrm{a}$ & $24,4 \mathrm{a}$ & $26,7 \mathrm{a}$ & $27,7 \mathrm{ab}$ & $28,8 \mathrm{a}$ & $29,6 a b$ & $30,3 b$ & $31,8 \mathrm{ab}$ & $32,4 \mathrm{ab}$ \\
\hline Orange & $19,5 \mathrm{~b}$ & $21,8 \mathrm{bc}$ & $23,9 b$ & $25,6 \mathrm{bc}$ & $26,5 \mathrm{~b}$ & $27,9 \mathrm{~b}$ & $28,7 \mathrm{~b}$ & $30,9 \mathrm{~b}$ & $31,4 \mathrm{~b}$ \\
\hline \multicolumn{10}{|l|}{ Solução } \\
\hline $\mathrm{S}_{1}$ & 20,1 & 22,7 & 25,1 & 25,9 & 27,4 & 28,8 & 29,8 & $31,1 \mathrm{~B}$ & 31,8 \\
\hline $\mathrm{S}_{2}$ & 20,8 & 22,9 & 25,2 & 27,2 & 28,4 & 29,8 & 31,0 & $32,9 \mathrm{~A}$ & 33,4 \\
\hline $\mathrm{C}$ & $* *$ & $* *$ & $* *$ & $* *$ & $* *$ & $* *$ & $* *$ & $*$ & $*$ \\
\hline S & $\mathrm{ns}$ & $\mathrm{ns}$ & $\mathrm{ns}$ & ns & ns & ns & $\mathrm{ns}$ & $*$ & $\mathrm{~ns}$ \\
\hline $\mathrm{C}^{*} \mathrm{~S}$ & $\mathrm{~ns}$ & $\mathrm{~ns}$ & $\mathrm{~ns}$ & $\mathrm{~ns}$ & $\mathrm{~ns}$ & $\mathrm{~ns}$ & $\mathrm{~ns}$ & $\mathrm{~ns}$ & $\mathrm{~ns}$ \\
\hline CV (\%) & 7,3 & 6,4 & 6,7 & 6,9 & 6,5 & 6,4 & 6,8 & 7,9 & 8,3 \\
\hline
\end{tabular}

Médias seguidas por letra diferente na coluna diferem entre si pelo teste de Tukey a $5 \%$, sendo minúsculas para cultivares e maiúsculas para soluções. C: cultivar. S: solução. $\mathrm{S}_{1}$ : $\mathrm{CE}$ de 0,92 e 1,07 $\mathrm{dS} \mathrm{m}^{-1} ; \mathrm{S}_{2}: 1,76$ e 2,04 dS m-1 (período vegetativo e reprodutivo, respectivamente). ns: não significativo ao nível de $5 \%$ de probabilidade. ${ }^{* *}$; ${ }^{*}$ significativo a 1 e $5 \%$ de probabilidade, respectivamente (means followed by the same lowercase letters in the column for cultivar and uppercase letters in the column for solution did not differ by the Tukey's test at 5\%. C: cultivar. S: solution. $\mathrm{S}_{1}$ : EC 0.92 and $1.07 \mathrm{dS} \mathrm{m}^{-1} ; \mathrm{S}_{2}: 1.76$ and $2.04 \mathrm{dS} \mathrm{m}^{-1}$ (vegetative and reproductive stages). ns: not significant at $5 \% ; *$ significant at $1 \%$; *significant at $5 \%$ ). 
Tabela 2. Área foliar (AF) e fitomassa seca total da parte aérea (FS) em cultivares de gérbera fertirrigadas com soluções nutritivas (foliar area (AF) and dry matter (FS) of gerbera cultivars fertigated with nutritive solution). Botucatu, UNESP, 2006.

\begin{tabular}{|c|c|c|c|c|c|}
\hline \multirow{3}{*}{ Cultivar } & \multicolumn{2}{|c|}{$\mathrm{AF}\left(\mathrm{cm}^{2}\right)$} & \multicolumn{3}{|c|}{ FS (g) } \\
\hline & \multirow{2}{*}{41 DAA } & \multirow{2}{*}{62 DAA } & \multirow{2}{*}{41 DAA } & \multicolumn{2}{|c|}{62 DAA } \\
\hline & & & & $S_{1}$ & $\mathbf{S}_{2}$ \\
\hline Cherry & 599,1 & $1116,6 \mathrm{ab}$ & $4,5 \mathrm{ab}$ & $12,0 \mathrm{abA}$ & $11,8 \mathrm{aA}$ \\
\hline Golden Yellow & 588,3 & $869,8 \mathrm{c}$ & $3,9 b$ & $10,4 \mathrm{bcA}$ & $11,9 \mathrm{aA}$ \\
\hline Salmon Rose & 708,7 & $1283,6 a$ & $5,2 \mathrm{a}$ & $13,4 \mathrm{aA}$ & $12,4 \mathrm{aA}$ \\
\hline Orange & 652,3 & $901,7 b c$ & $4,3 \mathrm{ab}$ & $8,8 \mathrm{cB}$ & $12,1 \mathrm{aA}$ \\
\hline \multicolumn{6}{|l|}{ Solução } \\
\hline $\mathrm{S}_{1}$ & 633,2 & 1034,7 & 4,3 & & \\
\hline $\mathrm{S}_{2}$ & 625,9 & 1051,1 & 4,7 & & \\
\hline $\mathrm{C}$ & $\mathrm{ns}$ & $* *$ & $*$ & \multicolumn{2}{|c|}{$*$} \\
\hline $\mathrm{S}$ & ns & $\mathrm{ns}$ & ns & \multicolumn{2}{|c|}{ ns } \\
\hline $\mathrm{C} * \mathrm{~S}$ & ns & ns & ns & \multicolumn{2}{|c|}{$*$} \\
\hline $\mathrm{CV}(\%)$ & 21,6 & 16,9 & 21,5 & \multicolumn{2}{|c|}{13,5} \\
\hline
\end{tabular}

Médias seguidas por letra diferente diferem entre si pelo teste Tukey a 5\%, sendo minúsculas na coluna entre cultivares e maiúsculas na linha entre soluções. C: cultivar; $S$ : solução; $S_{1}$ : $\mathrm{CE}$ de 0,92 e 1,07 $\mathrm{dS} \mathrm{m}^{-1} ; \mathrm{S}_{2}: 1,76$ e $2,04 \mathrm{dS} \mathrm{m}^{-1}$ (período vegetativo e reprodutivo, respectivamente); ns: não significativo ao nível de $5 \%$ de probabilidade; **;*significativo a $1 \mathrm{e}$ $5 \%$ de probabilidade, respectivamente; DAA: dias após aclimatação (means followed by the same lowercase letters in the column for cultivar and uppercase letters in the line for solution did not differ by the Tukey's test at 5\%; C: cultivar; S: solution; $\mathrm{S}_{1}$ : EC 0.92 and $1.07 \mathrm{dS}$ $\mathrm{m}^{-1} ; \mathrm{S}_{2}: 1.76$ and $2.04 \mathrm{dS} \mathrm{m}^{-1}$ (vegetative and reproductive stages); ns: not significant at 5\%; ** significant at $1 \%$; *significant at $5 \%$; DAA: days after acclimation).

$\left.\mathrm{dia}^{-1}\right)$, indicando que a cultura apresenta crescimento vegetativo constante ao longo do ciclo, mesmo quando a planta entra em estádio reprodutivo. Tendência semelhante foi constatada para a fitomassa seca da parte aérea (FS), a qual se desenvolveu de modo mais acentuado no terço final do ciclo da cultura, sugerindo que além das folhas, a emissão das hastes florais confere grande parte da FS. A constatação do maior crescimento no final do ciclo permite inferir que a demanda de nutrientes é superior nesse período, pois, segundo Orbes (2008) há um paralelismo entre a produção de fitomassa e o acúmulo de nutrientes.

Aos 41 DAA, observou-se que as cultivares Salmon Rose e Golden Yellow apresentaram a maior e menor FS, respectivamente (Tabela 2). Aos 62 DAA, quando houve efeito da interação entre cultivares e soluções nutritivas, esta tendência também foi observada na $S_{1}$. A cultivar Orange, quando conduzida com a $\mathrm{S}_{1}$, apresentou menor FS, possivelmente por esta solução fornecer menor quantidade de nutrientes, em especial o nitrogênio. $\mathrm{O} \mathrm{N}$ está pre- sente nos aminoácidos que formam as proteínas e na molécula de clorofila. A falta deste elemento acaba interferindo diretamente no processo fotossintético e reduzindo o crescimento vegetativo das plantas (Malavolta, 1980). Bellé (1998) encontrou respostas significativas para a FS de plantas de gérbera de vaso com aumento da concentração da adubação. Zheng et al. (2004) não constataram diferença na FS de gérbera de vaso com diferentes concentrações das soluções nutritivas aplicadas.

O tempo necessário para a produção (TP) foi semelhante para todas as cultivares (Tabela 3). Resultados discordantes foram encontrados por Singh \& Mandhar (2001), os quais concluíram que o florescimento precoce ou tardio é controlado por fatores genéticos (cultivares). Possivelmente o desbaste dos primeiros botões formados (BD), durante fases iniciais de produção, que é uma prática usual para induzir a formação de folhas, tenha contribuído para esta falta de significância, pois um número superior destes foi retirado de Cherry e Salmon Rose.
As cultivares próprias para cultivo em vaso foram selecionadas com base em características específicas, como a produção de inflorescências com hastes curtas, limitando a altura final da planta (AP). Em relação a esta variável, Salmon Rose apresentou-se superior e Orange inferior, com $32,8 \mathrm{~cm}$ e 28,2 $\mathrm{cm}$, respectivamente (Tabela 3 ), valores estes adequados segundo o padrão de comercialização, o qual preconiza plantas de no máximo de $50 \mathrm{~cm}$. Mota (2007) ao avaliar as cultivares Cherry, Golden Yellow e Salmon Rose sob diferentes níveis de $\mathrm{CE}$ da solução nutritiva, não registrou diferença na altura de planta entre as cultivares, com valores médios de $33 \mathrm{~cm}$.

As cultivares não diferiram em relação ao número de inflorescências (NI) (Tabela 3), concordando com resultados obtidos por Mota (2007). Diferença entre cultivares de gérbera de corte foi encontrada por Pisanu et al. (1994), Mascarini (1998) e Singh \& Mandhar (2001). O NI em plantas de vaso valoriza o produto final e pode ser decisivo na comercialização. Levando em consideração que a exigência do mercado para gérbera é de que o vaso tenha no mínimo dois botões florais abertos, observou-se em todas as cultivares, que essa exigência foi atendida.

O NI também pode exercer influência no ciclo da cultura, de modo que é possível atingir maior número de hastes florais, com um ciclo superior ao obtido neste experimento. A prática do desbaste dos botões florais torna possível o manejo da cultura, a fim de obter um número maior de hastes florais, entretanto com o aumento do ciclo e consequentemente no custo de produção.

$\mathrm{O}$ diâmetro de inflorescência (DI) apresentou-se superior na cultivar Salmon Rose, e inferior na cultivar Golden Yellow (Tabela 3), sendo uma característica influenciada principalmente pelo potencial genético. Vários trabalhos relatam a diferença de DI entre cultivares de gérbera de corte (Goldsberry \& Lang, 1987; Singh \& Mandhar, 2001; Sonneveld \& Voogt, 1983).

As cultivares apresentaram comportamento diferenciado em cada solução nutritiva para o diâmetro de haste (DH), com diferença na $\mathrm{S}_{1}$, onde o maior 
Tabela 3. Botões florais desbastados (BD), tempo para produção (TP), altura de planta (AP), número de inflorescências (NI), diâmetro de inflorescência (DI) e diâmetro de haste (DH) das plantas em ponto de comercialização, em função das cultivares e soluções nutritivas (removed floral buds (BD), time to produce (TP), height of plant (AP), number of inflorescences (NI), inflorescence diameter (DI) and stick diameter (DH) at the commercialization stage of gerbera depending on cultivar and nutritive solution). Botucatu, UNESP, 2006.

\begin{tabular}{lccccccc}
\hline \multirow{2}{*}{ Cultivar } & BD & TP(DAA) & AP (cm) & NI & DI (mm) & \multicolumn{2}{c}{ DH (mm) } \\
\cline { 6 - 8 } & & & & & & $\mathbf{S}_{1}$ & $\mathbf{S}_{2}$ \\
\hline Cherry & $19,9 \mathrm{a}$ & 63,0 & $29,7 \mathrm{ab}$ & 3,2 & $92,8 \mathrm{ab}$ & $5,5 \mathrm{bA}$ & $5,7 \mathrm{aA}$ \\
Golden Yellow & $9,7 \mathrm{~b}$ & 60,5 & $29,9 \mathrm{ab}$ & 2,7 & $87,7 \mathrm{~b}$ & $5,3 \mathrm{bB}$ & $6,3 \mathrm{aA}$ \\
Salmon Rose & $22,8 \mathrm{a}$ & 62,3 & $32,8 \mathrm{a}$ & 2,4 & $103,1 \mathrm{a}$ & $6,1 \mathrm{abA}$ & $5,6 \mathrm{aA}$ \\
Orange & $6,9 \mathrm{~b}$ & 60,0 & $28,2 \mathrm{~b}$ & 3,0 & $90,8 \mathrm{ab}$ & $6,7 \mathrm{aA}$ & $6,1 \mathrm{aA}$ \\
\hline Solução & & & & & & & \\
\hline $\mathrm{S}_{1}$ & 14,9 & 60,8 & 30,7 & 2,6 & 95,7 & & \\
$\mathrm{~S}_{2}$ & 14,8 & 62,0 & 29,6 & 3,0 & 91,5 & & \\
\hline $\mathrm{C}$ & $* *$ & $\mathrm{~ns}$ & $*$ & $\mathrm{~ns}$ & $*$ & & $*$ \\
$\mathrm{~S}$ & $\mathrm{~ns}$ & $\mathrm{~ns}$ & $\mathrm{~ns}$ & $\mathrm{~ns}$ & $\mathrm{~ns}$ & & $\mathrm{~ns}$ \\
$\mathrm{C} * \mathrm{~S}$ & $\mathrm{~ns}$ & $\mathrm{~ns}$ & $\mathrm{~ns}$ & $\mathrm{~ns}$ & $\mathrm{~ns}$ & & $*$ \\
\hline $\mathrm{CV}(\%)$ & 18,4 & 5,5 & 9,6 & 42,8 & 11,9 & & 10,4 \\
\hline
\end{tabular}

Médias seguidas por letra diferente diferem entre si pelo teste Tukey a 5\%, sendo minúsculas na coluna entre cultivares e maiúsculas na linha entre soluções. C: cultivar; S: solução; $\mathrm{S}_{1}$ : CE de 0,92 e 1,07 dS m ${ }^{-1} ; \mathrm{S}_{2}: 1,76$ e 2,04 dS m-1 (período vegetativo e reprodutivo, respectivamente); ns: não significativo ao nível de $5 \%$ de probabilidade; $* * ; *$ significativo a 5 e $1 \%$ de probabilidade, respectivamente; DAA: dias após aclimatação (means followed by the same lowercase letters in the same column for cultivar and uppercase letters in the same line for solution did not differ by the Tukey's test at 5\%. C: cultivar; S: solution; $\mathrm{S}_{1}$ : EC 0.92 and $1.07 \mathrm{dS} \mathrm{m}^{-1} ; \mathrm{S}_{2}: 1.76$ and $2.04 \mathrm{dS} \mathrm{m}^{-1}$ (vegetative and reproductive stages); ns: not significant; **significant at 1\%; *significant at 5\%; DAA: days after acclimation).

diâmetro foi encontrado em Orange, sem diferir significativamente de Salmon Rose. A cultivar Golden Yellow apresentou menor diâmetro quando conduzido com a $\mathrm{S}_{1}$, comparada à $\mathrm{S}_{2}$, resultado este não observado para as demais cultivares (Tabela 3). Em trabalhos realizados com gérbera de corte, Fakhri et al. (1995) verificaram que o diâmetro de haste não foi afetado pela cultivar, entretanto Singh \& Mandhar (2001) registraram diferentes resultados.

A maior CE fornecida pela solução $\mathrm{S}_{2}$, não representou problema de estresse osmótico às plantas, pois segundo Sonneveld (2000), o efeito mais comum no crescimento da planta em condições de estresse osmótico na zona da raiz é a redução da fitomassa, frequentemente coincidindo com o decréscimo da área foliar, altura e espessura da haste.

É adequado que haja relação entre altura de planta e diâmetro da haste, pois hastes altas e finas tendem a ter reduzida sustentação, tornando-se mais suscetíveis à quebra e diminuindo a qualidade estética ou até impossibilitando a comercialização. Salmon Rose apresentou maior altura de planta, porém compensado pelo diâmetro de haste, superior, tendo assim a estabilidade necessária. A maior formação de parte aérea foi registrada para as cultivares Cherry e Salmon Rose e menor para Golden Yellow e Orange, com qualidade produtiva satisfatória nas duas soluções nutritivas.

\section{REFERÊNCIAS}

BARBOSA JG; MARTINEZ HEP; KÄMPF AN. 1999. Acúmulo de macronutrientes em plantas de crisântemo sob cultivo hidropônico em argila expandida para flor de corte. Pesquisa Agropecuária Brasileira 34: 593-601.

BELLÉ S. 1998. Sistemas de irrigação e concentrações de adubação complementar na produção de Gerbera jamesonii cv 1187 em vaso. Porto Alegre: UFRGS. 122p (Tese doutorado).

CHUNG YM; KIM HA; KIM KY; PARK SW; YI YB; LEE JH; KNOW OC. 2001. Morphological characteristics and genetic variation of gerbera (Gerbera hybrida Hort.). Journal of Plant Biotechnology 3: 145-149.

FAKHRI MN; MALOUPAE; GERASOPOULOS D. 1995. Effects of substrate and frequency of irrigation on yield and quality of three Gerbera jamesonii cultivars. Acta Horticulturae 408: 41-45.

FANELA TLM; MOTA PRD; VILLAS BÖAS RL; LUDWIG F; FERNANDES DM. 2006. Influência de diferentes níveis de tensão de água na cultura da gérbera desenvolvida em substrato. In: MOSTRA CIENTÍFICA EM CIÊNCIAS AGRÁRIAS, 2.; MOSTRA CIENTÍFICA DA FMVZ, 10.; REUNIÃO
CIENTÍFICA EM CIÊNCIAS AGRÁRIAS DO LAGEADO, 13. Anais... Botucatu: UNESP (CD-ROM).

FERREIRA DF. 2000. Análises estatísticas por meio do Sisvar para Windows versão 4.0. In: REUNIÃO ANUAL DA REGIÃO BRASILEIRA DA SOCIEDADE INTERNACIONAL DE BIOMETRIA, 45. Anais... São Carlos: UFSCar. p.255-258.

GOLDSBERRY KL; LANG RC. 1987. Response of gerbera to root zone heating in soil and gravel substrates. HortScience 22: 595-597.

INFOAGRO. 2005, 20 de junho. El cultivo de la gerbera. Disponível em http://www. infoagro. com.htm/

LIN WC; FRENCH CJ. 1985. Effects of supplementary lighting and soil warming on flowering of three gerbera cultivars. HortScience 20: 271-273.

MALAVOLTA E. 1980. Elementos de nutrição mineral de plantas. São Paulo: Agronômica Ceres. 253p.

MASCARINI L. 1998. Gerbera cultivation in growing media. Horticultura Internacional 6: 86-88.

MOTA PRD. 2004. Niveis de condutividade elétrica da solução do substrato em crisântemo de vaso, em ambiente protegido. Botucatu: UNESP-FCA. 82p (Tese mestrado).

MOTA PRD. 2007. Aplicação via fertirrigação de soluções com diferentes condutividades elétricas para produção de gérbera (Gerbera jamesonii L.) sob ambiente protegido. Botucatu: UNESP-FCA. 149p (Tese doutorado).

ORBES MY. 2008. Marcha de absorção de nutrientes, produção e qualidade de hastes florais de plantas de tango (Solidago 
canadensis L.). Viçosa: UFLA. 75p (Tese mestrado).

PARADISO R; DE PASCALE S; APREA F; BARBIERI G. 2003. Effect of electrical conductivity levels of nutrient solution on growth, gas exchanges and yield of two gerbera cultivars in soilless system. Acta Horticulturae 609: 165-171.

PISANU AB; CARLETTI MG; LEONI S. 1994. Gerbera jamesonii cultivation with different inert substrates. Acta Horticulturae 361:
590-602.

ROGERS MN; TJIA BO. 1990. Gerbera production. Portland: Timber Press. 116p.

SINGH KP; MANDHAR SC. 2001. Performance of exotic cultivars of gerbera (Gerbera jamesonii) under low cost naturally ventilated greenhouse environment. Indian Journal of Agricultural Sciences 71: 244-248.

SONNEVELD C; VOOGT W. 1983. Studies on the salt tolerance of some flower crops grown under glass. Plant and Soil 74: 41-52.
SONNEVELD C. 2000. Effects of salinity on substrate grown vegetables and ornamentals in greenhouse horticulture. Netherlands: Wageningen Universitiet. 149p (Tese doutorado).

ZHENG Y; GRAHAM T; RICHARD S; DIXON M. 2004. Potted gerbera production in a subirrigation system using low-concentration nutrient solutions. HortScience 39: $1283-$ 1286. 